\title{
HACIA UNA PERSPECTIVA DE GÉNERO EN LA EVALUACIÓN DE LA CALIDAD DE LA ATENCIÓN EN LOS SERVICIOS DE SALUD REPRODUCTIVA.
}

Mira. Mario de los Angeles Torres Lagunas:

*Protesora de Carrero Asociada "B" del Sistema Universidad Abienta ENEO-UNAM.

RESUMEN.

La necesidad de integrar la perspectiva de género en las políticas y programas gubernamentales de nuestro pais, ha sido reconocida a partir de los compromisos acordados en la Conferencia Internacional sobre Población y Desarrollo efectuada en El Cairo (1994) y de la Cuarta Conferencia Internacional de la Mujer celebrada en Pekín (1995), de las cuales han derivado recomendacionies por parte de Organización Mundial de la Salud (OMS) y de la Organización Panamericana de la Salud (OPS) a los países miembros, para que estos se comprometan a reconocer y corregir las inequidades de género que vulneran el ejercicio del derecho a la salud entre los habitantes de los Estados Miembros, apoyados en tres pilares conceptuales: equidad, género y participación democrática.

En México existen pocas investigaciones que evalúan la calidad de la atención en los servicios de salud sexual y reproductiva desde la perspectiva de género. Por lo tanto, es necesario realizar nuevos estudios con este enfoque e implementar nuevas formas de evaluar la calidad de la atención en estos servicios, que incluyan indicadores construidos desde la perspectiva de género, que consideren metodologías cualitativas, que superen el carácter administrativo y normativo centrado en la estructura y en los resultados y que su orientación no solamente tenga que ver con metas demográficas relativas a la efectividad en el uso de métodos anticonceptivos y en niveles de fecundidad alcanzados. Estrategias evaluativas más humanas que analicen la dimensión social de la calidad desde el ejercicio del derecho a la salud en lo general y de los derechos sexuales y reproductivos en lo particular considerando a la persona como un ser integral.

PALABRAS CLAVE: Evaluación de la calidad, Calidad de la atención, perspectiva de género, salud sexual y reproductiva.

\section{ABSTRACT}

The need for integrating gender perspective into the government politics and programs of our country has been acknowledged since the commitments agreed at the Population and Development International Conference carried out in El Cairo (1994) and in the Fourth Women International Conference in Pekin (1995), which have derived recommendations from the World Health Organization $(W H O)$ and the Pan-American Health Organizotion (PAHO) for the member countries to accomplish such commitments.

In Mexica, there is little research evaluating sexual and reproductive health services attention quality from a gender perspective. Therefore, it is necessary $t$ carry out new studies using this focus and to implement new ways for evaluating care quality in such services that include indicators built up from the gender view, and taking in account qualitative methodologies, surpassing the managerial and normative character focused on outcomes and whose orientation not only has to do with the demographic goals relative to their effectiveness in the use of contraceptive methods and on fertility levels reached. Also needed are mare human assessment strategies for analyzing quality social dimension starting from the exercise of health rights in general, and from the sexual and reproductive rights in particular, and that consider the person as an integral being.

KEY WORDS: Attention quality - quality evaluation - gender perspective - sexual and reproductive health. 


\section{ANTECEDENTES}

El interés por el estudio de la calidad de la atención desde una perspectiva de género inicia en América Latina por la década de los años ochenta con los estudios del grupo de investigación de Maria Isabel Matamala de la Red de Salud por las Mujeres Latinoamericanas y del Caribe (RSMLAC) y de algunas otras académicas feministas procedentes de Organizaciones No Gubernamentales (ONG), quienes fueron pioneras en incluir otras categorías en el análisis de la calidad de la atención además de las ya tradicionales referidas por Avedis Donabedian, relativas al monitoreo de la estructura, del proceso y de los resultados.

Fué hasta la década de los años noventas que ha sido considerada esta temática a partir de los compromisos acordados en la Conferencia Internacional Sobre Población y Desarrollo celebrada en El Cairo (1994), en la IV Conferencia Mundial sobre la Mujer celebrada en Beijing (1995) y teniendo como referente fundamental la Convención Sobre la Eliminación de Todas Formas de Discriminación Contra la Mujer (CEDAW) efectuada en 1979. Dichas Conferencias han derivado entre otros los siguientes compromisos:

a) Establecer las condiciones propicias para asegurar la cobertura, utilización y calidad de los servicios de salud integrales.

b) Reconocer los derechos reproductivos.

c) Establecer las condiciones propicias para asegurar la salud sexual y reproductiva para que se eleve la calidad de vida.

d) Eliminar la brecha de desigualdad, al promover la emancipación, el empoderamiento de las mujeres y la equidad de género, asi como asegurar el acceso universal y salvaguardar el respeto de los derechos reproductivos, reconociendo la desigual posición y la condición de las mujeres en el mundo.

A partir de estas Conferencias, surgieron algunos estudios relativos a la calidad de la atención en los servicios de salud reproductiva que consideraban otras dimensiones socioculturales que influyen en la calidad de la atención de las personas usuarias. En este contexto, aparecen los estudios de Elsa Gómez de la Organización Panamericana de la Salud (OPS), de Hilda Reyes de la ONG Afluentes y del Instituto Nacional de Perinatología (INPer), de Monserrat Salas de la ONG Salud Integral para la Mujer (SIPAM), de Mónica Jasis, Juan Guillermo Figueroa y Roberto Castro de El Colegio de México (COLMEX) y los estudios de Gisela Espinosa, Lorena Paz, Gustavo Leal y Carolina Martinez y Dora Cardaci, de la Universidad Autónoma Metropolitana de Xochimilco (UAM) entre otras y otros investigadores que han sido pioneras/os en la formulación de marcos conceptuales para analizar la calidad de la atención con perspectiva de género, además, de los siguientes modelos de evaluación de la calidad con esta perspectiva propuestos por las siguientes investigadoras:

- Judith Bruce del Population Council (1998). Su modelo comprende 6 elementos para evaluar la calidad: elección de métodos, información a los usuarios/as, competencia técnica de las y los proveedores, relaciones interpersonales, continuidad y seguimiento y constelación adecuada de servicios.

- María Isabel Matamala (2001) Su modelo comprende 6 elementos para evaluar la calidad: Dignificación de usuarias, conciencia, ejercicio y respeto de derechos humanos, competencia técnica, vínculo interpersonal y potenciación de comportamientos favorables.

Hilda Reyes (2001) Ella crea un sistema de monitoreo y evaluación de los servicios de salud sustentado en indicadores y estándares de calidad que se centran en la atención a las/os usuarias/os desde una perspectiva de género.

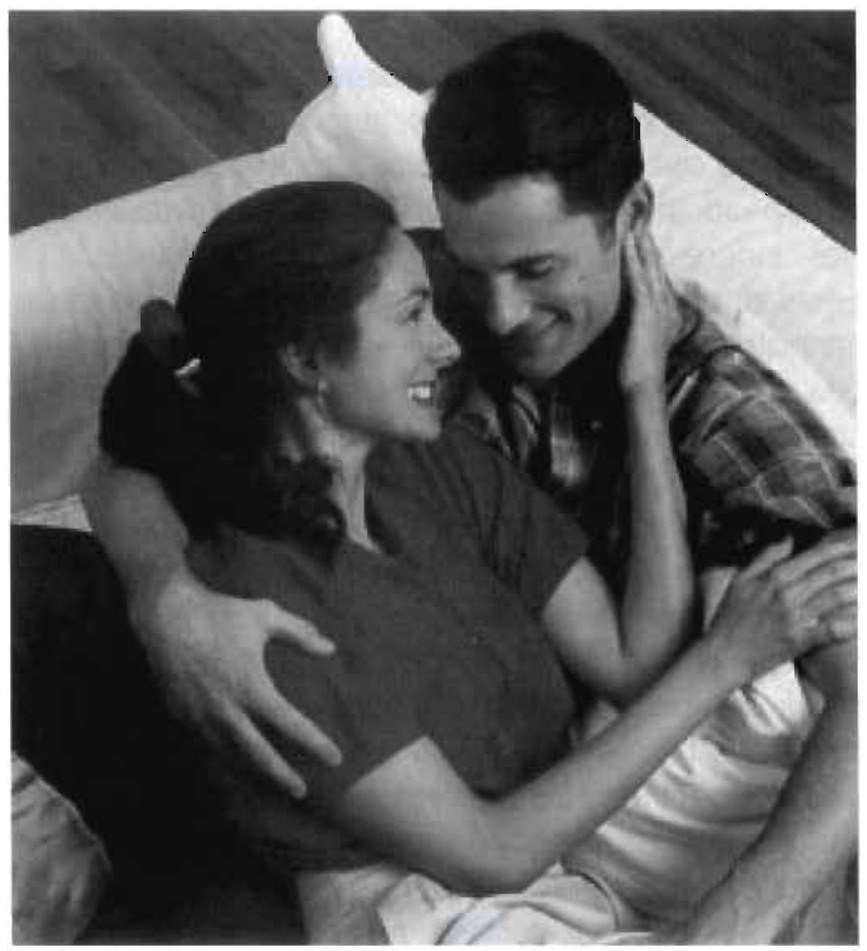




\section{Conceptos básicos relativos a los servicios de salud de calidad con perspectiva de género.}

\section{Servicios de salud de calidad con perspectiva de género.}

Para la ONG mexicana "Salud Integral para la Mujer" (SIPAM), los servicios de salud de calidad con perspectiva de género dirigidos a las mujeres son aquellos que responden a las necesidades de salud integral de las usuarias, con alternativas técnicas de alto nivel y respetuosas de su dignidad; que garantizan la resolución del motivo de la consulta en el momento oportuno y a un costo accesible; que toman en cuenta el conocimiento que tienen las mujeres sobre sus procesos de salud; que propician su autoestima, su autonomía, percepción y ejercicio del derecho a la salud y a decidir; y que promueven la participación conciente y equitativa de los hombres y mujeres en los procesos de salud sexual y reproductiva.

\section{Calidad de la atención a la Salud Reproductiva desde la perspectiva de género.}

Es aquella que provee una atención integral a las personas usuarias sustentada en principios éticos y de equidad. Esta considera las diferencias socioculturales, favorece la autoestima, el respeto mutuo, el trato digno y la conciencia y ejercicio de los derechos humanos, en particular de derechos sexuales y reproductivos.

\section{Equidad en salud.}

La equidad en salud es la consideración de las disparidades evitables en salud y sus determinantes entre grupos humanos con diferentes niveles de privilegio social. La noción adoptada por la OMS/OPS es la de "desigualdades innecesarias, evitables y además injustas". La Equidad no es lo mismo que igualdad, la igualdad es un conocimiento empírico, la equidad representa un imperativo de carácter ético asociado con principios de justicia social y de derechos humanos. Es el resultado de analizar y eliminar todo tipo de discriminación que afecte directa e indirectamente la salud de las mujeres.

\section{Salud integral de la mujer.}

Desde una perspectiva feminista, este término incluye no sólo los aspectos biológicos relacionados con el ciclo de vida de la mujer y los relativos a la reproducción (que son los que usualmente son considerados cuando se aborda la salud de las mujeres), si no de manera fundamental los aspectos socioculturales que tienen que ver

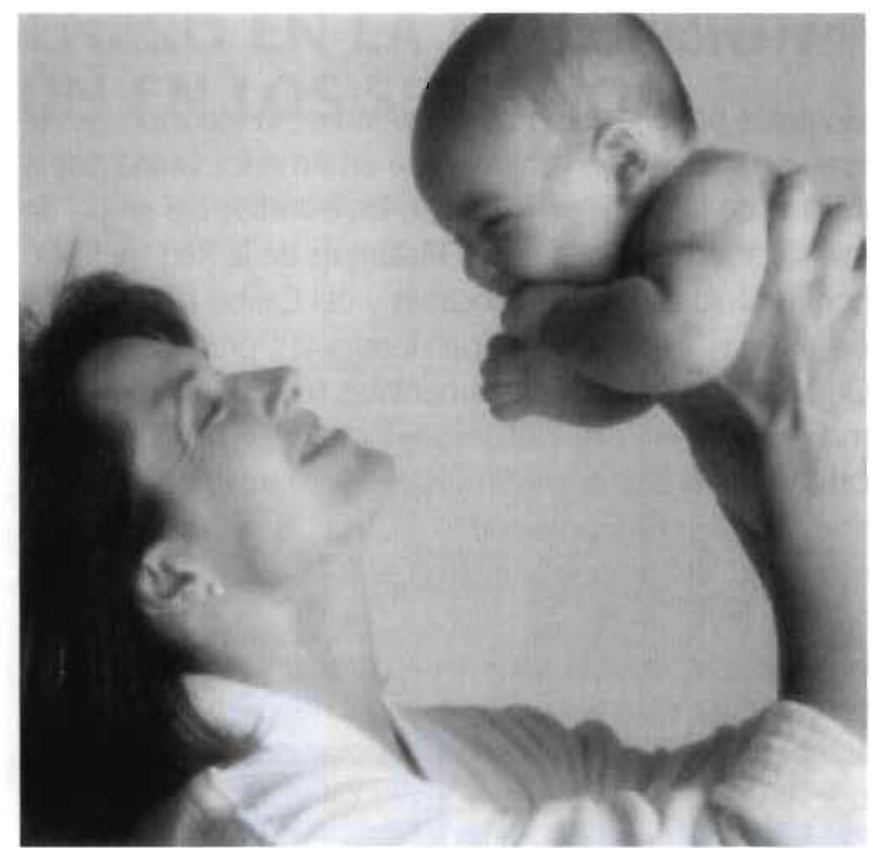

con sus relaciones interpersonales, con sus sentimientos, con sus necesidades, con sus vivencias con el ser "para otros" y no para sí, con el asumir estereotipos asociados con las "mujeres" y con el desigual acceso a los bienes y servicios.

\section{Calidad y salud reproductiva.}

La calidad de los servicios de salud impacta a mujeres y a hombres que acuden como usuarias y usuarios; sin embargo, cuando se trata de servicios de salud reproductiva, la insuficiente calidad de la atención recae particularmente en las mujeres, pues son estas las que mayoritariamente acuden a solicitar estos servicios.

En México la igualdad de derechos de mujeres y hombres está reconocida en el artículo 40 de la Constitución. Sin embargo, las prácticas discriminatorias por razón de género persisten en muchas organizaciones. El espacio de la salud es un sitio donde se generan relaciones asimétricas de poder, entre quienes proveen y quienes reciben el servicio. Es en estos lugares en donde independientemente del sexo de las personas involucradas en la atención, quien provee el servicio se comporta de manera "masculina" y quien lo recibe lo hace de manera "femerina", asumiendo asi roles y estereotipos androcéntricos (Es cuando se considera al hombre como el paradigma del ser humano. $Y$ el hombre es visto como lo esencial o central de cualquier actividad) que nuestra sociedad ha asociado con cada sexo: seguridad, poder, autoridad, fuerza, racionalidad, objetividad, iniciativa, etcétera para lo "masculino" y sumisión, abnegación, paciencia, subjetividad, emotividad, inseguridad para lo "femenino". 
Las instituciones de salud son estructuras patriarcales (sistemas familiares, sociales, ideológicos y politicos en los cuales los hombres por medio de la fuerza directa, los rituales, la tradición, las leyes, el lenguaje, las costumbres, la educación y especialmente la división del trabajo determinan cuál es o no el papel que las mujeres deben interpretar a fin de mantenerlas siempre sometidas a la autoridad del hombre) diferenciadas por condición de género, son el sitio donde es común observar situaciones que generan diversas formas de discriminación que se traducen en actos de violencia, tales como: El maltrato, la descalificación y la indiferencia hacia las/los usuarias/os, el no respeto a su privacidad y pudor, el lenguaje técnico, discriminatorio poco entendible e infantilista, la desinformación en general de que son objeto, la subvaloración a sus opiniones y comentarios, la poca disponibilidad a ponerles atención, a mirarles a los ojos, a escucharlas/os, los largos tiempos de espera a los que se les somete, las dificultades de acceso, la falta de continuidad y seguimiento a la atención por sobresaturación de citas, las iatrogenias y complicaciones por problemas de competencia técnica, los problemas éticos, y el deterioro de la atención que se refleja en una percepción general de mala calidad en la atención

Por qué evaluar la calidad desde la perspectiva de género.

La necesidad de analizar desde una perspectiva de género la calidad de la atención en los servicios de salud reproductiva responde entonces, al interés de monitorear

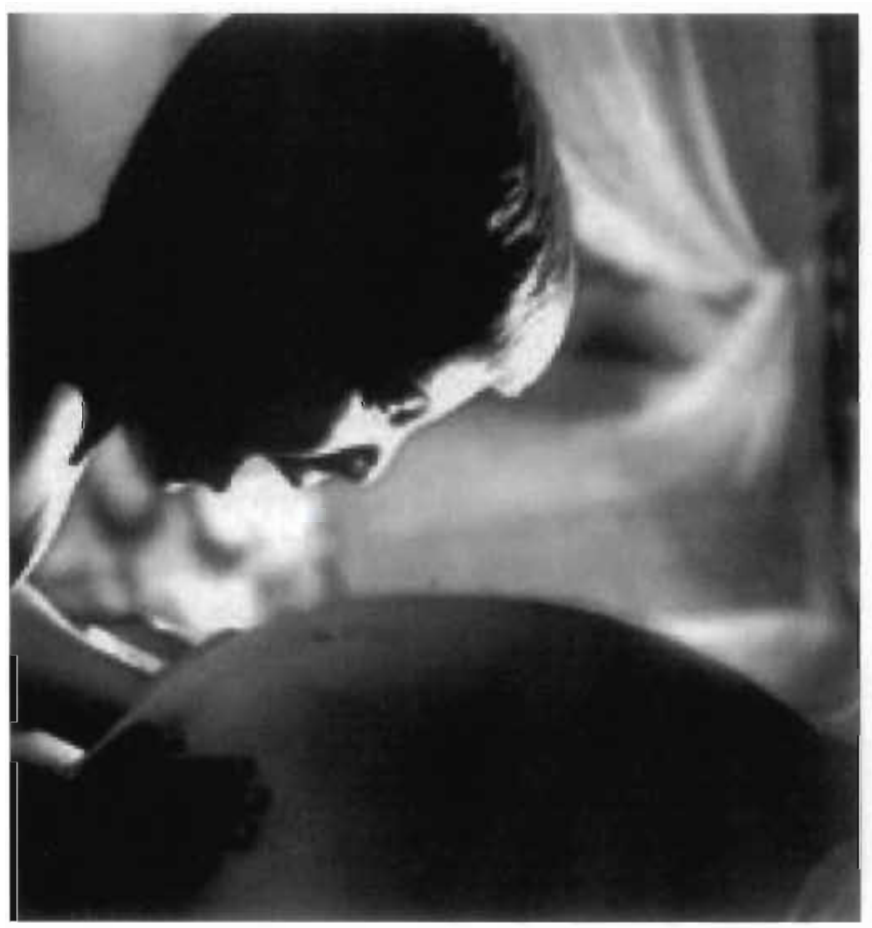

y evaluar dichos servicios considerando indicadores socioculturales construídos desde esta perspectiva. Indicadores que estén centrados en las necesidades de las personas, que reflejen las condiciones en que se presenta la relación intersubjetiva en la cual tiene lugar el acto clínico, que consideren las relaciones interpersonales entre usuarias y proveedores como uno de los elementos centrales que muestran la calidad de la atención y que permiten identificar y evidenciar las inequidades y asimetrías por razón de género que se viven de manera cotidia na en la provisión de servicios de salud reproductiva en las instituciones de salud. Dichas inequidades violan los derechos humanos de las personas en tanto son usuarias de dichos servicios.

La perspectiva de género se constituye así en una herramienta metodológica útil para identificar estas inequidades y genera a una visión crítica del proceso en que se da la relación terapéutica, permitiendo analizar cómo y por qué las mujeres vivimos diversas condiciones de inequidad sólo por ser mujeres. Analizar la calidad de la atención desde una perspectiva de género incluye la visión crítica no sólo de los aspectos de estructura y resultados que es donde comúnmente se realizan las evaluaciones, sino también es analizar en qué condiciones se da el proceso de la atención, cómo se da el acceso a los servicios, cómo se dan las relaciones interpersonales entre usuarias y proveedores, el grado de conocimiento de los derechos y responsabilidades de las usuarias/os, el estado de la competencia técnica de las y los proveedores, la información que se brinda a unas y otros, el identificar cómo y quién toma las decisiones en salud reproductiva, etc. entre otras categorias.

\section{Una atención de calidad en los servicios de salud reproductiva implica:}

- Respetar los derechos humanos de las personas usuarias/os de los servicios de salud reproductiva, en particular sus derechos sexuales y reproductivos y sus derechos como usuarias/os de los servicios de salud. Brindar un trato respetuoso y digno.

- Responder a las necesidades de salud integral de las usuarias/os, con alternativas técnicas de alto nivel.

- Favorecer la equidad entre los géneros en la provisión de servicios de salud sexual y reproductiva, corresponsabilizando al varón en los aspectos relacionados con la reproducción.

- Tomar en cuenta el conocimiento que tienen las mujeres/hombres sobre sus procesos de salud.

- Garantizar la resolución del motivo de consulta en el momento oportuno y a un costo accesible. 
Utilizar un lenguaje no discriminatorio, sin calificativos. Brindar información apropiada y oportuna.

Alentar los procesos de empoderamiento y autoestima, en las y los usuarios.

Formación y capacitación en estudios de género.

- Existencia de normas y programas que incorporen la perspectiva de género.

- Contar con presupuestos sensibles al género.

- Tener estadísticas y sistemas de registro desagregados por sexo.

\section{REFLEXIONES FINALES}

Una atención con perspectiva de género es más humana, considera la salud integral de las personas y los aspectos subjetivos que se dan en una relación terapéutica donde intervienen mujeres y hombres como usuarias/os y proveedoras/es.

La perspectiva de género nos permite tener una visión integral de la realidad para hacer visibles las discriminaciones e inequidades y de otros factores que conforman la calidad. No se trata sólo de superar indicadores numéricos o el logro de objetivos y metas programáticas evaluables. Se trata de ver la atención y el cuidado a la salud como algo integral a partir de encontrar los factores que expliquen las insatisfacciones y las deficiencias en la atención por razón de género en un marco de derechos humanos.

La relación proveedora/or - usuaria/o debe sustentarse en principios éticos básicos, como son el de respeto de su individualidad y autonomía como personas, el de beneficencia, referente a no causar perjuicios y dar mayores beneficios posibles y el de justicia que está relacionado con los derechos de las personas en un plano de equidad.

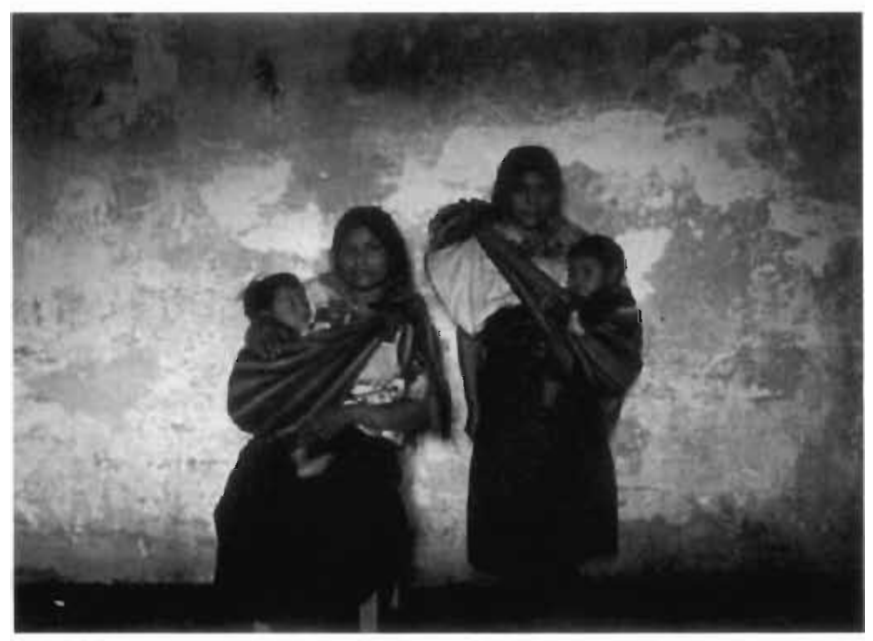

\section{REFEREIVCIAS BIBLIOGRÁFICAS.}

Alatorre, Edda. "La atención gineco-obstétrica en el marco de los Derechos Humanos". En: Memoria de la Reunión Nacional sobre Derechos Humanos de la Mujer. CNDH. México, D.E. 2002, p. 2.

Facio, Alda. El principio de igualdad ante la ley. Manuscrito de la Directora del Programa, Mujer, Justicia y Género del Instituto Latinoamericano para la prevención del Delito - ILANUD, Costa Rica, 1997, p. ì

Bruce, Judith. "Elementos fundamentales de la calidad de la atención : un marco conceptual sencillo.". Ed. Population Council, Documentos de trabajo No. 21, Nueva York, U.S.A. 1998, 41 pp.

CDHNU. Declaración de los derechos Humanos desde una perspectiva de género. Aportes al 50 aniversario de la Declaración Universal de los Derechos Humanos. Ed. CDHNU, Documento No. E/CN.4/1998/NGO/3, Ginebra, 1998, p. 1

Gómez, Elsa. "Equidad de género y salud. Mitos y realidades "; en: Revista Mujer y Salud. Red de salud de las mujeres latinoamericanas y del caribe. $3 / 2000$, p. 10-17

Gómez, Elsa. "Género, equidad y acceso a los servicios de salud: una aproximación empírica" en: Revista Panam Salud Pública/Pan Am J Healt! $11(15-6), 2002$ p. 454.

Langer, Ana y Rafael Lozano. "Condición de la mujer y salud"; en : Figueroa, Juan (Coordinador) La condición de la mujer en el espacio de la salud. Ed. El Colegio de México. 1998, p 36

Matamala, Ma. Isabel. "Prólogo La ciudadanía, el control ciudadano y la calidad. Porque la calidad no es sólo una cuestión de tecnología y humanidad, es también una cuestión político-social". en: SALAS, Monserrat. (Coordinadora). Calidad de la atención con perspectiva de género. Ed. SIPAM, Plaza y Valdez Editores. México, 2001, p. 16.

Matamala, Ma. Isabel y Cols. "Calidad de la atenciôn. Género, isalud reproductiva de las mujeres?". Santiago de Chile, reproducción América. Editora. Maria Isabel Matamala (COMUSAMS-ACHNU), Santiago de Chile, 1995, p. 1 ?

Mejía, M. Consuelo. "Legitimidad de los derechos sexuales y reproductivos" en: Violencia sobre la salud de las mujeres. Roberto, Uribe \& Deborah Billings (Editores), Ed. Femego, IPAS México, A.C. México, 2003, pp. 124-128.

Reyes, Hilda y Colș. "Modelo de mejora continua de la calidad de la atención a la salud sexual y reproductiva con perspectiva de género" en: Calidad de la atención con perspectiva de género. Ed. SIPAM, Plaza y valdez. Editores. México, 2001, pp. 61-97.

Reyes, Hilda. "La calidad de la atención a la salud sexual y reproductiva: un compromiso para lograr una maternidad sin riesgos": En el libro Maternidad sin riesgos en Puebla. Ed. Comité Promotor por una Maternidad sin Riesgos. México, 2001, pp.23-38.

Salas, Monserrat: (Coordinadora). Calidad de la atención con perspectiva de género. Ed. SIPAM, Plaza y Valdez Editores. México, 2001, p. $17-17$.

Salas, Monserrat. "Una vez más: ¿Cuál es la perspectiva de género en la calidad de la atención médica" en: Calidad de lá atención en salud sexual y reproductiva. Ed. DGSR de la SS y COLMEX, Comité Promotor por una Maternidad sin Riesgos. Segundo Coloquio sobre Calidad de Atención, efectuado los dias 22 y 23 de octubre de 1996 en México, 1997, p. 56

\section{DIRECCIÓN PARA CORRESPONDENCIA:}

Maria de los Angeles Torres Lagunas: angeles_torres2000@yahoo.com.mx 